\title{
Spine maturation and pruning during development: Cadherin/Catenin complexes come to help
}

\author{
QIAN Cheng ${ }^{1,2} \&$ LI YuLong ${ }^{1 *}$ \\ ${ }^{1}$ State Key Laboratory of Membrane Biology, Peking University School of Life Sciences, PKU-IDG/McGovern Institute for Brain Research, \\ and Peking-Tsinghua Center for Life Sciences, Beijing 100871, China; \\ ${ }^{2}$ School of Life Sciences, PTN Joint Graduate Program, Tsinghua University, Beijing 100084, China
}

Received August 25, 2015; accepted September 1, 2015

Citation: Qian C, Li YL. Spine maturation and pruning during development: Cadherin/Catenin complexes come to help. Sci China Life Sci, 2015, 58: 929-930, doi: 10.1007/s11427-015-4933-1

Structural and functional alterations of synaptic connections underlay experience-dependent neuronal plasticity and are crucial for many brain functions, including learning and memory. Their defects, on the other hand, have been associated with neurological disorders such as autism. Although the molecular mechanism underlying long-term functional alterations of synaptic connections, including LTP and LTD, are becoming increasingly clear, the molecular mechanism for structural plasticity, such as spine pruning, remains largely unknown, especially in vivo.

In a recent research article published in Cell, the research group led by Dr. Xiang Yu from the Institute of Neuroscience, Chinese Academy of Sciences, discovered that the Cadherin/Catenin cell adhesion complex plays a central role in coordinating dendritic spine pruning and maturation during experience-dependent plasticity in vivo [1]. This work significantly extends our current knowledge of the molecular mechanism underlying structural synaptic plasticity.

Dr. Xiang Yu and her colleagues first established a robust in vivo paradigm for investigating activity-dependent synapse remodeling. They focused on the primary somatosensory cortex $(\mathrm{S} 1 \mathrm{BF})$, a brain region that receives and processes tactile sensory information during development. They found, intriguingly, that enriching the environment of mice from birth, by increasing their sensory inputs and their social interactions, robustly accelerated the process of spine

*Corresponding author (email: yulong@gmail.com) pruning during the critical developmental period of 1 to 3 months in the S1BF. Additionally, they noticed that the degree of spine maturation, specifically the proportion of mature mushroom and stubby spines over immature thin and branched spines, showed an inverse correlation with spine density. Importantly, deprivation of sensory inputs to S1BF by whisker trimming prevented both spine pruning and maturation, demonstrating the instrumental role of neural activity in regulating structural spine plasticity.

To explore the possible underlying molecular mechanism, Yu's group focused on important cell adhesion molecules enriched on synapses, together with their associated partners. They found that conditional removal of $\beta$-catenin, which forms a cell adhesion complex with $\mathrm{N}$-cadherin, led to defects in spine pruning and maturation in a cell-autonomous manner in S1BF. Consistent with the crucial roles of Catenin/Cadherin complexes, overexpression of a dominant negative form of N-cadherin, or knockout of $c d f$, which encodes $\alpha \mathrm{N}$-catenin, another critical component of the Catenin/Cadherin complex, both led to similar defects. Thus, the Cadherin/Catenin complex is necessary for spine pruning and maturation in vivo (Figure 1).

To investigate how the Cadherin/Catenin complex exerts its local action, Yu's group cleverly localized N-cadherin locally on target spines in cultured neurons and observed its effects on both the target spine and neighboring spines. They achieved this by applying inert micro-beads coated with $\mathrm{N}$-cadherin-Fc, which only binds to and activates $\mathrm{N}$ - 


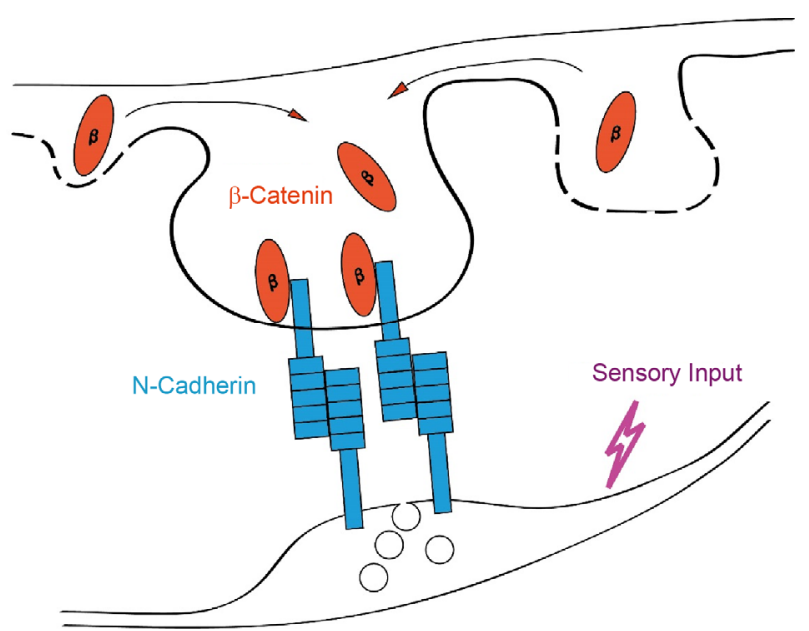

Figure 1 (color online) Activity-dependent inter-spine competition of limited Cadherin/Catenin complexes is crucial for coordinated spine pruning and maturation.

cadherins in spines which it directly contacts. By timelapse imaging, they first observed that the bead-contacting spines grew larger, while the neighboring non-contacting spines shrank. They further found that more Cadherin/Catenin complexes were recruited in bead-contacting spines than the ones nearby. They went additional steps to test these results in the in vivo setting by establishing a smart spine competition assay using transgenic mice expressing a stabilized form of $\beta$-catenin. They observed similar effects as in culture neurons. Taken together, these data indicated that spines compete for limiting resources of Cadherin/Catenin complexes for their local stabilization (Figure 1). Moreover, the function of the Cadherin/Catenin complex is specific, because enhancing the action of the Neuroligin /Neurexin complex, another important pair of synaptic adhesion molecules, only led to a global increase of overall spine growth, rather than inter-spine competition.

Do sensory inputs or neuronal activity regulate spine fate by engaging Cadherin/Catenin complexes? To test this possibility, the authors introduced channelrhodopsins (CHR) into presynaptic neurons-a powerful optogenetic approach that enables light- and cell-specific activation of neurons. They found that postsynaptic spines in contact with presynaptic CHR-positive axons grew larger and accumulated more $\beta$-catenin upon photostimulation, while their neighboring non-contacting spines shrank and lost their $\beta$-catenin. Knocking down $\beta$-catenin by RNAi abolished this phenomenon, demonstrating the essential role of the Cadherin/Catenin complex in this process.

Together, the combined in vitro and in vivo experimental results allowed the authors to convincingly establish a causal link between inter-spines competition for Cadherin/ Catenin complexes and spine pruning/maturation (Figure 1). Similar to many great research articles, the current paper also raises many interesting unanswered questions.

Firstly, how does sensory experience or neuronal activity affect the Cadherin/Catenin complex and the subsequent spine pruning? Erin Schuman's group previously reported that activation of NMDA receptors may lead to slowing down of the plasma membrane $\mathrm{N}$-cadherin internalization [2]. Thus, it would be interesting to directly test the role of calcium in synapse-specific stabilization of $\mathrm{N}$-cadherin. Secondly, $\beta$-catenin was previously reported to mediate dendritic morphogenesis through $\mathrm{N}$-cadherin-dependent adhesion and sometimes increased Wnt release [3]. How does the nervous system coordinate dendritic growth vs. spine pruning/maturation, e.g. how does $\beta$-catenin selectively prune spines without affecting dendritic morphogenesis? Is local Wnt signaling involved in spine pruning/maturation, too? Thirdly, how does the Cadherin/Catenin complex coordinate with other signaling pathways important for synapse formation or maturation? For example, a few studies have demonstrated that important synaptic adhesion molecules, including the Neuroligin/ Neurexin complex, promoted spinogenesis [4]. Do Cadherin/Catenin and Neuroligin/Neurexin complexes act totally independently or they converge to some common downstream effectors? If these signaling pathways converge, what are their shared targets? How do these different pathways coordinate in a spatiotemporal manner?

Taken together, the pioneering finding of the Cadherin/Catenin complex's role in spine pruning and maturation from Dr. Xiang Yu and her colleagues leads us to a deeper understanding of the molecular underpinning of activity-dependent structural remodeling of synapses. Further exploration of these molecular mechanisms may provide more insights into potential therapeutic targets for intervention in various neurological disorders.

1 Bian WJ, Miao WY, He SJ, Qiu Z, Yu X. Coordinated spine pruning and maturation mediated by inter-spine competition for Cadherin/ Catenin complexes. Cell, 2015, 162: 808-822

2 Tai CY, Mysore SP, Chiu C, Schuman EM. Activity-regulated N-cadherin endocytosis. Neuron, 2007, 54: 771-785

$3 \mathrm{Yu} \mathrm{X}$, Malenka RC. $\beta$-catenin is critical for dendritic morphogenesis. Nat Neurosci, 2003, 6: 1169-1177

4 Chih B, Engelman H, Scheiffele P. Control of excitatory and inhibitory synapse formation by neuroligins. Science, 2005, 307: $1324-1328$

Open Access This article is distributed under the terms of the Creative Commons Attribution License which permits any use, distribution, and reproduction in any medium, provided the original author(s) and source are credited. 